\title{
XXXVIII. The travelling cyclone
}

\section{Lord Rayleigh O.M. F.R.S.}

To cite this article: Lord Rayleigh O.M. F.R.S. (1919) XXXVIII. The travelling cyclone, Philosophical Magazine Series 6, 38:225, 420-424, DOI: 10.1080/14786440908635965

To link to this article: http://dx.doi.org/10.1080/14786440908635965

$$
\text { 册 Published online: } 08 \text { Apr } 2009 .
$$

Submit your article to this journal 준

Џ Article views: 5

Q View related articles $\asymp$ 
XXXVIII. The Travelling Cyclone.

By the late Lord Rayleigu, O.M., F.R.S."

[Note.-The concluding paragraphs of this paper were dictated by my father only five days before his deatl. The proofs therefore were not revised by him. The figure was unfortunately lost in the post, and I have redrawn it from the indications given in the text.-RAYLEIGH.]

NE of the most important questions in meteorology is the constitution of the travelling cyclone, for cyclones usually travel. Sir N. Shaw $\dagger$ says that "a velocity of. 20 metres/second (44 iniles per hour) for the centre of a cyclonic depression is large but not unknown; a velocity of less than 10 metres/second may be regarded as smaller than the average. A tropical revolving storm usually travels at about 4 motres/second." He treats in detail the comparatively simple case where the motion (relative to the ground) is that of a solid body, whether a simple rotation, or such a rotation combined with a uniform translation; and he draws important conclusions which must find approximate application to travelling cyclones in general. One objection to regarding this case as typical is that, unless the rotating area is infinite, a discontinuity is involved at the distance from the centre where it terminates. A more general treatment is desirable, which shall allow us to suppose a gradual falling off of rotation as the distance from the centre increases; and I propose to take up the general problem in two dimensions, starting from the usual Eulerian equations as referred to uniformly rotating axes $\ddagger$. The density $(\rho)$ is supposed to be constant, and gravity can be disregarded. In the usual notation we have

where

$$
\begin{aligned}
& \frac{1 d p}{\rho d x}=\omega^{2} x+2 \omega v-\frac{\mathrm{D} u}{\mathrm{D} t}, \quad . \quad . \quad . \\
& \frac{1 d p}{\rho d y}=\omega^{2} y-2 \omega u-\frac{\mathrm{D} x}{\mathrm{D} t}, \quad . \quad . \quad .
\end{aligned}
$$

$$
\mathrm{D} / \mathrm{D} t=d / d t+u d / d x+v d / d y . \quad . \quad . \quad .
$$

Here $x, y$ are the coordinates of a point, referred to axes

* Communicated by the Author.

+ 'Manual of Meteorology;' Purt iv. p. 121, Cambridge, 1919.

† Lamb's 'Hydrodynamics,' \$207, 1916. 
revolving uniformly in the plane $x y$ with angular velocity $\omega^{*}$, $u$ and $v$ are the components of relative velocity of the fluid in the directions of the revolving axes, that is the components of wind. We have now to define the motion for which we wish to determine the balancing pressures.

We contemplate a motion (relatively to the ground) of rotation about a centre $\mathrm{C}$, fig. 1 , situated on the axis of $x$,

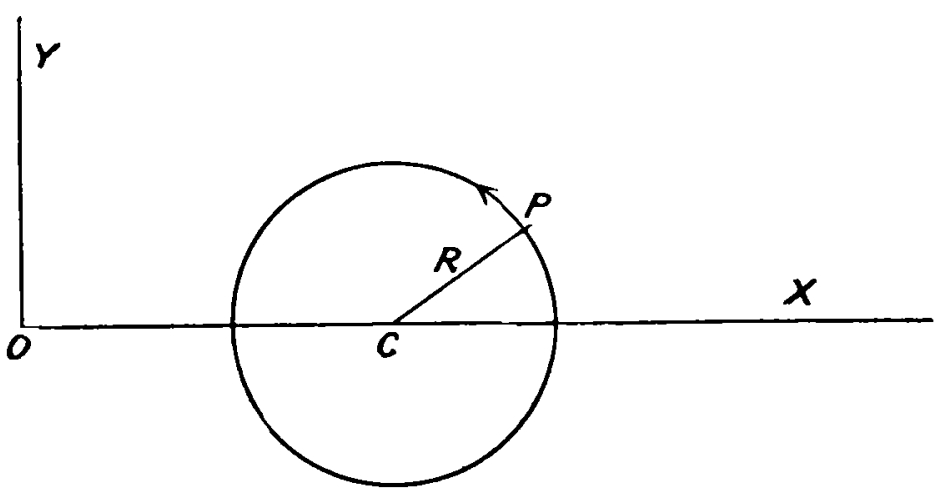

the successive rings $P$ at distance $R$ from $C$ revolving with an angular velocity $\zeta$, which may be a function of $R$. And upon this is to be superposed a uniform velocity of translation U, parallel to $x$ and carrying everything forward. If initially $\mathrm{C}$ be at $\mathrm{O}$, the fixed origin, its distance from $\mathrm{O}$ along $\mathrm{O} x$ at time $t$ will be Ut. Thus

$$
u=\mathrm{U}-\zeta y, \quad v=\zeta(x-\mathrm{U} t), . . .
$$

$\zeta$ being a known function of $\mathrm{R}$, where

$$
\mathrm{R}^{2}=y^{2}+(x-\mathrm{U} t)^{2}=y^{2}+\mathrm{X}^{2} .
$$

These equations give $u$ and $v$ in terms of the coordinates and of the time, and the values are to be introduced into (1) and (2). From the manner in which $x$ and $t$ enter (representing a uniform translation of the entire system) it is evident that $d / d t=-\mathrm{U} d / d x$. We have

$$
\begin{aligned}
& \frac{d u}{d x}=-\frac{\zeta^{\prime} \mathrm{X} y}{\mathrm{R}}, \quad \frac{d u}{d y}=-\zeta-\frac{\zeta^{\prime} y^{2}}{\mathrm{R}} \\
& \frac{d v}{d x}=\zeta+\frac{\zeta^{\prime} \mathrm{X}^{2}}{\mathrm{R}}, \quad \frac{d v}{d y}=\frac{\zeta^{\prime} \mathrm{X} y}{\mathrm{R}},
\end{aligned}
$$

* In the application to a part of the eartli's atmosphere, $\omega$ is the earth's angular velocity nultiplied by the sine of the latitude. 
$\zeta^{\prime}$ being written for $d \zeta / d \mathrm{R}$; and

$$
\begin{aligned}
& \frac{\mathrm{D} u}{\mathrm{D} t}=\frac{\zeta^{\prime} \mathrm{UX} y}{\mathrm{R}}-u \frac{\zeta^{\prime} \mathrm{X} y}{\mathrm{R}}-v\left(\zeta+\frac{\zeta^{\prime} y^{2}}{\mathrm{R}}\right)=-\zeta^{2} \mathrm{X} \\
& \frac{\mathrm{D} v}{\mathrm{D} t}=-\mathrm{U}\left(\zeta+\frac{\zeta^{\prime} \mathrm{X}^{2}}{\mathrm{R}}\right)+u\left(\zeta+\frac{\zeta^{\prime} \mathrm{X}^{2}}{\mathrm{R}}\right)+v \frac{\zeta^{\prime} \mathrm{X} y}{\mathrm{R}}=-\zeta^{2} y .
\end{aligned}
$$

Hence

$\frac{1}{\rho} \frac{d p}{d x}=\omega^{2} x+2 \omega \zeta \mathrm{X}+\zeta^{2} \mathrm{X}, \frac{1}{\rho} \frac{d p}{d y}=\omega^{2} y-2 \omega\left(\mathrm{U}-\zeta_{y}\right)+\zeta^{2} y,$.

and on integration

$$
\frac{p}{\rho}=\frac{1}{2} \omega^{2}\left(x^{2}+y^{2}\right)-2 \omega \mathrm{U} y+\int\left(2 a \zeta+\zeta^{2}\right) \mathrm{R} d \mathrm{R} .
$$

As might have beon expected, the last term in (7) is the same function of $R$ as when $U=0$, but $R$ itself is now a function of $\mathrm{U}$ and $t$.

In the case considered by Sir N. Shaw, $\zeta$ is constant and may be removed from under the integral sign. Thus

$$
\stackrel{p}{\rho}={ }_{2}^{1} \omega^{2}\left(x^{2}+y^{2}\right)-2 \omega \mathrm{U} y+\left(\omega \zeta+\frac{1}{2} \zeta^{2}\right)\left\{y^{2}+(x-\mathrm{U} t)^{2}\right\} .
$$

If $\mathrm{U}=0, \mathrm{R}^{2}$ identifies itself with $x^{2}+y^{2}$, and we get

$$
p / \rho=\frac{1}{2}(\omega+\zeta)^{2}\left(x^{2}+y^{2}\right) . \quad \text {. . . . . }
$$

A constant as regards $x$ and $y$, which might be a function of $t$, may be added in (8) and (9).

We see that if $\omega+\zeta=0$, that is if the original terrestrial rotation is annulled by the superposed rotation, $p$ is constant, the whole fluid mass being in fact at rest. It was for the purpose of this verification that the terms in $\omega^{2}$ were retained. We may now omit them as representing a pressure independent of the motion under consideration. In the strictly two-dimensional problem there is a pressure increasing outwards due to "centrifugal force." In the application to the earth's atmosphere, this pressure is balanced by a component of gravity connected with the earth's ellipticity. Thus in Shaw's case we have

$$
\begin{aligned}
& p \\
& \rho
\end{aligned}=\text { Const. }+\left(\omega \zeta+\frac{1}{2} \zeta^{2}\right)\left\{\left(y-\begin{array}{c}
\omega \mathrm{U} \\
\omega \zeta+\frac{1}{2} \zeta^{2}
\end{array}\right)^{2}+(x-\mathrm{U} t)^{2}\right\},
$$


showing that the field of pressure, though still circular, is no longer centred at $\mathrm{O}$ as when $\mathrm{U}=0$, or even at $\mathrm{C}$, where $x=\mathrm{U} t, y=0$, but is displaced sideways to the point where $x=\mathrm{U} t, y=\omega \mathrm{U} /\left(\omega \zeta+\frac{1}{2} \zeta^{2}\right)$. Shaw calls this the dynamic centre; it is the point which is conspicuons on the weather map as the centre of the system of circular isolars.

As a case where the circular molion diminishes to nothing as we go outwards, let us nuw suppose that $\zeta=Z^{-\mathrm{R}^{2} / \mathrm{x}^{2}}$, falling off slowly at first but afterwards with great rapidity. We have

$\int_{0}^{\mathrm{R}} \zeta \mathrm{R} d \mathrm{R}=\frac{1}{2} Z a^{2}\left(1-e^{-\mathrm{R} 2 / \alpha^{2}}\right), \quad \int_{0}^{\mathrm{R}} \zeta^{2} \mathrm{R} d \mathrm{R}=\frac{1}{4} \mathrm{Z}^{2} a^{2}\left(1-e^{-2 \mathrm{R}^{2} / a^{2}}\right) ;$ and thus from (7)

$$
\frac{p}{\rho}=\text { Const. }-2 \omega \mathrm{U} y-\frac{1}{2} a^{2}\left(\mathrm{Z} e^{-\mathrm{R} 2 / a^{2}}+2 \omega\right)^{2}, \quad .
$$

where, as usual, $\mathrm{R}^{2}=y^{2}+(x-\mathrm{U} t)^{2}$.

May 17 th.

The completion of this paper was interrupted by illness.

The two-dimensional solution requires a ceiling, as well as a floor, to take the pressure. In the absence of a ceiling we must introduce gravity, and since in the supposed motion no part of the fluid is vertically accelerated, the third equation of motion gives simply

$$
\frac{p}{\rho}=\text { const. }-g z \text {. }
$$

Thus (10) is altered merely by the addition of the term $-g z$.

I had supposed too that the solution would remain substantially unaltered even though $\rho$ were variable as a function of $p$. But these conclusions seem to be at variance with those put forward by Dr. Jeffreys in the January No. of the Philosophical Magazine. I am not able to pursue the comparison at present.

June 25th, 1919.

[The following note has been contributed by Sir Joseph Larmor.-EDs.

This paper was left incomplete on Lord Rayleigh's decease on June 30 . It may therefore be permissible to direct 
attention to its main conclusion from another.aspect, by way of paraphrase. 'T'wo questions are involved. If a vortical system can persist at rest, in an atmosphere rolating with the Earth, can it also persist, slightly modifed, with a translatory velocity $U$ ? And if so, how will the distribution of pressure in it be modifierl? The equations of fluid motion relative to the ground are (1) and (2); in them the last terms $\mathrm{D} u / \mathrm{D} t$ and $\mathrm{D} v / \mathrm{D} t$ express the components of relative acceleration, and these are clearly the centrif ugal accelerations $-\zeta^{2} \mathrm{X},-\zeta^{2} y$ in the relative orbits assumed to be circular, as found analytically lower down. On substituting these values, the equations give for $\delta p$ an exact differential form which is integrated in (7); therefore a moditied motion is possible, and the fiust question is answered in the affirmative, in agreement so far with fact *. The displacement of the pressure-system due to the progressive motion is then examined for two special cases by the formula (10) and (11), showing also general agreement with fact as regards displacement of the centre of the vortex. But the value of $U$ is not determined by these considerations, which refer to frictionless fluid. When viscosity in the fluid is taken into account, the general argument seems to remain applicable; for the velocity of convection $U$, being uniform, will not modify the viscous stresses. But, in any case, internal viscosity is negligible in meteorological problems. It is the friction aguinst land or ocean, introducing turbulence which spreads upward, that disturbs and ultimately destroys the cyclonic system; and the bigh degree of permanence of the type of motion seems to permit that also to be left out of account. As remarked in the postscript, the changes of pressure arising from convection involve changes of density, which will modify the motion, but perhaps slightly. There does not seem to be definite discordance with Dr. Jeffreys' detailed discussion.]

- The conditions of stability for flow of liquid with varying vorticity had been considered in a series of papers, for which reference nay be made to the section Hydrodynamics of the catalogue appended to Lord Rayleigh's 'Collected Papers,' vol. iv. 